\title{
An efficient method for solving sound propagation in range-dependent ocean waveguides was found by researchers from Chinese Academy of Sciences
}

The coupled normal mode method is a powerful approach for solving range-dependent propagation problems in underwater acoustics. An
important area of study is to improve stability and efficiency so as to be able to deal with complex scenarios in a realistic environment.
Professor Luo Wenyu and his group from the State Key Laboratory of Acoustics, Institute of Acoustics, Chinese Academy of Sciences, set
out to tackle this problem. After several years of innovative research, they have developed an accurate, efficient, and numerically stable
coupled normal mode method to solve the range-dependent propagation problem. Their work, entitled "A numerically stable coupled-mode
formulation for acoustic propagation in range-dependent waveguides", was published in Science China Physics, Mechanics \& Astronomy, 2012, Vol. 55 (4).

Underwater sound propagation in range-dependent waveguides is critical to many studies and applications in the area of underwater acoustics. Neglect of waveguide range-dependence may lead to significant prediction errors. A number of approaches have been developed for solving this problem. Despite significant recent advances, problems such as intensive computation and instability remain unsolved. Therefore, the need for developing new approaches with better efficiency, stability, and accuracy is urgent.

In the method proposed by Luo et al., the direct global matrix (DGM) approach is applied. As is well-established, the primary advantage of the DGM approach is that it gives numerically stable solutions when there is evanescence across layers, and it does this without special numerical treatment. Therefore, the proposed method is unconditionally stable. Furthermore, by introducing appropriately normalized range solutions, the overflow problem inherent in certain existing models is eliminated. In addition, general source conditions were put forward, which significantly extends the applicability of the proposed model compared to existing models.

To validate a range-dependent model, we have the following possible methods: comparison with analytical solutions, checking energy conservation and reciprocity and inter-model comparison. The proposed method is validated by comparison with the analytical solution to an ideal wedge benchmark problem. Here, a range-dependent problem involving a wedge-shaped waveguide with pressure-release boundaries is analyzed. Since sound propagation towards the wedge apex will be completely backscattered due to perfectly reflecting boundaries, this test problem is an ideal benchmark for a full two-way solution to the wave equation. The comparison indicates that the proposed model is highly accurate and numerically stable (as shown in Figure 1). Furthermore, this method provides high computational efficiency. The execution time for the proposed model is less than $10 \%$ of that of the COUPLE model, which is a widely used coupled normal mode model. Note that although an ideal waveguide problem is used to validate the proposed method, the formulation presented also applies to realistic waveguides with penetrable bottoms and/or depth-variant sound speed profiles in water.

Implementation and promotion of this work will contribute significantly to the study of underwater sound propagation.

The proposed method proves to be accurate, efficient, and numerically stable. The researchers suggest their work be extended and applied to the study of three-dimensional effects, for instance, the horizontal refraction that is present in complex environments. This will have significant impact on the study of three-dimensional underwater sound propagation.
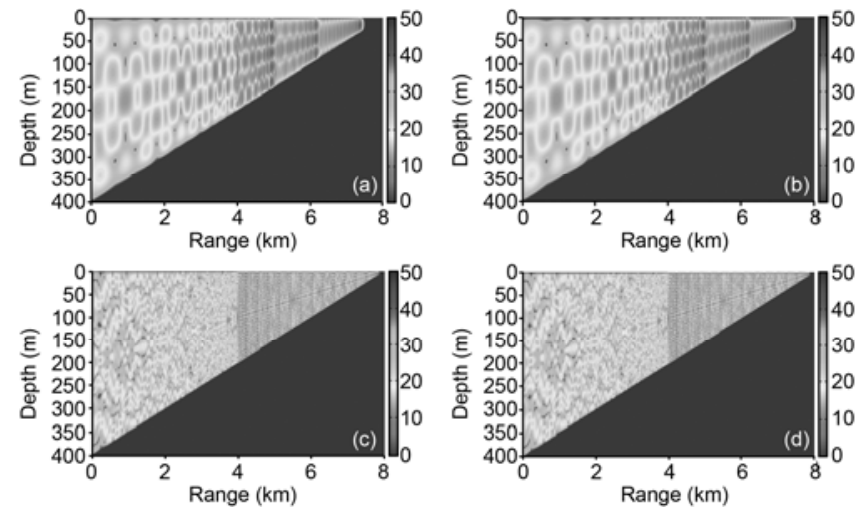

Figure 1 Transmission loss in the water column. The source is located at a range of $4 \mathrm{~km}$ and a depth of $100 \mathrm{~m}$ by (a) analytical solution at $25 \mathrm{~Hz}$; (b) present model at $25 \mathrm{~Hz}$; (c) analytical solution at $100 \mathrm{~Hz}$; (d) present model at $100 \mathrm{~Hz}$.

This work was supported by the National Natural Science Foundation of China (10734100 and 11125420) and the Knowledge Innovation Program of Chinese Academy of Sciences.

See the article: Luo W Y, Yang C M, Qin J X, et al. A numerically stable coupled-mode formulation for acoustic propagation in range-dependent waveguides. Sci China Phys Mech Astron, 2012, 55: 572-588

Open Access This article is distributed under the terms of the Creative Commons Attribution License which permits any use, distribution, and reproduction in any medium, provided the original author(s) and source are credited. 